Japan. J. Math.

Vol. 10, No. 1, 1984

\title{
Equivariant transfer for finite G-coverings
}

\author{
By Hisashi Fukagawa
}

(Received June 13, 1983)

\section{Introduction}

Transfers $t(p): \Sigma^{\infty}\left(Y_{+}\right) \rightarrow \Sigma^{\infty}\left(X_{+}\right)$for finite coverings $p: X \rightarrow Y$ were studied by D. S. Kahn, S. B. Priddy [6], [7] and by F. W. Roush [13]. Roush proved the uniqueness of the transfer satisfying certain axioms.

Let $G$ be a finite group throughout the present work in which we define equivariant transfers for finite $G$-coverings and prove their uniqueness. Our approach is, in a sense, parallel to that of Roush [13] even though we encounter several problems of different nature coming from $G$-actions.

In $\S 1$ we discuss the equivariant version of the little cubes operads defined by M. Boardman and M. Vogt [4] and studied by P. May [9]. We define $G$-little cubes operads $\mathscr{C}_{\infty}(k ; G)$ (Definition 1.1$)$ which is used to construct the equivariant transfer. $G \times \widetilde{S}_{k}$ acts on $\mathscr{C}_{\infty}(k ; G)$ and for a subgroup $\tilde{H}$ of $G \times \widetilde{S}_{k}$, the fixed-point set $\mathscr{C}_{\infty}(k ; G)^{\tilde{H}}$ is contractible if $\tilde{H}=\{(h, \tau(h)) \mid h \in H\}$ for a homomorphism $\tau: H \rightarrow \mathfrak{S}_{k}, H \leqq G$, and is empty otherwise. Therefore $\mathscr{C}_{\infty}(k ; G) / \mathfrak{S}_{k}$ is a classifying space of $\mathfrak{S}_{k}$-bundles with $G$-action. In $\S 2$ we define the equivariant transfer (Definition 2.2) and the equivariant wreath operation (Definition 2.5), and investigate the relation between them. Let $\Gamma$ be a subgroup of $\widetilde{S}_{k}$ and $\operatorname{Cov}_{G}^{\Gamma}$ be the category of $k$-fold $G$-coverings $p$ : $(X, A) \rightarrow(Y, B)$ with structure group $\Gamma$ and pullback diagrams as morphisms. A generalized equivariant transfer $t$ is a correspondence of $p \in \operatorname{Cov}_{G}^{\Gamma}$ to $t(p) \epsilon$ $[Y \mid B, Q(X \mid A)]^{G}$ which is natural with respect to pullback diagrams (Definition 2.2). We postulate two axioms (T1) and (T2) for generalized equivariant transfers similar to the axioms of Roush (Definition 2.2 and [13], Chapter VI, Theorem 1B), and define an equivariant transfer as generalized equivariant transfer satisfying these axioms. Our main theorem is

TheOREM 2.3. The equivariant transfer on $\operatorname{Cov}_{G}^{\Gamma}$ exists uniquely.

To prove this theorem we take a way parallel to the arguments of Roush. We define an equivariant wreath operation $w$ as $G$-homotopy classes $w(X) \in\left[\mathscr{C}_{\infty}(k ; G)_{+} \bigwedge_{\Gamma} X^{k}, Q_{G}(X)\right]^{G}$ which is natural with respect to based $G$ maps of $X$. We show that generalized equivariant transfers correspond to 
equivariant wreath operations bijectively (Lemma 2.8) and rewrite the axioms (T1) and (T2) on $t$ as the axioms (W1) and (W2) on $w$ (Lemma 2.9, 2.10). In $\S 3$ we show equivariant wreath operations satisfying (W1) and (W2) correspond to equivariant stable wreath operations (Definition 3.1) bijectively and prove that the equivariant stable wreath operation exists uniquely in Proposition 3.5. The proof of this proposition is the most complicated part in this work in comparison with the non equivariant case of Roush.

In $\S 4$ we show that our equivariant transfer $t(p)$ is an adjoint of the equivariant Becker-Gottlieb transfer $\tau(p)$ for a finite $G$-covering over a finite $G$-complex (Nishida [12]), and also the induced homomorphism in $K$-theory is equal to the equivariant Atiyah transfer.

The author is grateful to Professors Shôrô Araki and Yasumasa Hirashima for their kind advices and encouragements.

\section{§1. $G$-little cubes operads}

We denote by $\rho$ the complex regular representation of $G$, by $D \rho$ its unit disk, by $S \rho$ its unit sphere and put $\stackrel{\circ}{D} \rho=D \rho-S \rho$.

Definition 1.1. A G-little $n \rho$-cube is a linear embedding $f$ of $\left(\dot{D}_{\rho} \rho\right)^{n}$ in $\left(\check{D}_{\rho}\right)^{n}$ such that $f=f_{1} \times \cdots \times f_{n}$ where $f_{i}: \check{D}_{\rho} \rho \rightarrow \check{D} \rho$ is a map $f_{i}(v)=x_{i}+\varepsilon_{i} v, x_{i} \in$ $\stackrel{\circ}{D} \rho, \varepsilon_{i}>0, v \in \check{D}_{\rho} \rho$.

Define $\mathscr{C}_{n}(k ; G)$ to be the set of those $k$-tuples $\left(c_{1}, \cdots, c_{k}\right)$ of $G$-little $n \rho$ cubes such that the images of $c_{i}$ are pairwise disjoint. We regard $\left(c_{1}, \cdots, c_{k}\right)$ as a map from $\left(\dot{D}_{\rho}\right)^{n} \amalg \cdots \amalg\left(\grave{D}_{\rho}\right)^{n}$, $k$-times, to $\left(\dot{D}_{\rho}\right)^{n}$, and topologise $\mathscr{C}_{n}(k ; G)$ as a subspace of the space of all continuous functions $\left(D_{\rho}\right)^{n} \amalg \cdots \amalg\left(D_{\rho}\right)^{n} \rightarrow$ $\left(\grave{D}_{\rho}\right)^{n}$. Define the left $G$-action and the right $\widetilde{S}_{k}$-action on $\dot{\mathscr{C}}_{n}(k ; G)$ as follows:

$$
\begin{aligned}
& \left(c_{1}, \cdots, c_{k}\right) \sigma=\left(c_{\sigma(1)}, \cdots, c_{\sigma(k)}\right), \\
& g\left(c_{1}, \cdots, c_{k}\right)=\left(g c_{1}, \cdots, g c_{k}\right), \quad g \in G,
\end{aligned}
$$

where $\widetilde{S}_{k}$ is the $k$-th symmetric group and $\left(g c_{i}\right)(v)=g\left(c_{i}\left(g^{-1} v\right)\right)$. Then $(g c) \sigma=$ $g(c \sigma)$ and we regard $\mathscr{C}_{n}(k ; G)$ as a left $G \times \widetilde{S}_{k}$-space by $(g, \sigma) c=g c \sigma^{-1}$.

Let us choose a non zero element $a \in\left(\dot{D}_{\rho}\right)^{G}$. There is an injection $\mathscr{C}_{n}(k ; G) \rightarrow\left(\stackrel{D}{D} \rho^{2 n}\right)^{2 n k}$ defined by

$$
\left(c_{1}, \cdots, c_{k}\right) \rightarrow\left(c_{1}(0, \cdots, 0), c_{1}(a, \cdots, a), \cdots, c_{k}(0, \cdots, 0), c_{k}(a, \cdots, a)\right) .
$$

This is a $G$-map and the space $\mathscr{C}_{n}(k ; G)$ can be regarded as a $G$-subspace of $\left(\check{D}_{\rho}\right)^{2 n k}$ by this injection.

Let $F(X ; k)$ be the configuration space $F(X ; k)=\left\{\left(x_{1}, \cdots, x_{k}\right) \in X^{k} \mid x_{i} \neq\right.$ 
$\left.x_{j}(i \neq j)\right\}$ for a $G$-space $X$. $\widetilde{\Im}_{k}$ acts on $F(X ; k)$ from the right by permutations of coordinates and $G$ acts on it from the left by the diagonal action. We regard $F(X ; k)$ as a $G \times \widetilde{S}_{k}$-space by $(g, \sigma) y=g y \sigma^{-1}$ for $g \in G, \sigma \in \mathfrak{S}_{k}$ and $y \in$ $F(X ; k)$.

LEMMA 1.2. The space $\mathscr{C}_{n}(k ; G)$ has the same $G \times \widetilde{S}_{k}$-homotopy type as $F\left(C[G]^{n} ; k\right)$, where $C[G]$ is the complex group ring of $G$.

Proof. Because of $C[G] \approx{ }_{G} \dot{D}_{\rho}$ we can replace $C[G]^{n}$ by $\left(\check{D}_{\rho}\right)^{n}$. Let $\phi$ : $\mathscr{C}_{n}(k ; G) \rightarrow F\left(\left(\check{D}_{\rho}\right)^{n} ; k\right)$ be the $G \times \widetilde{S}_{k}-\operatorname{map} \phi\left(\left(c_{1}, \cdots, c_{k}\right)\right)=\left(c_{1}(0), \cdots, c_{k}(0)\right)$. We define the $G \times \Im_{k}$-homotopy inverse $\psi$ of $\phi$ as follows. For $c=\left(c_{1}, \cdots, c_{k}\right) \in$

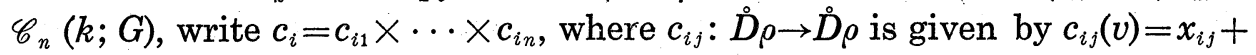
$\varepsilon_{i j} v$. We say this $c$ is the equidiameter of radius $d$ if $\varepsilon_{i j}=d$ for all $i$ and $j$. Let $\psi(b)=c$ for $b \in F\left(\left(\dot{D}_{\rho}\right)^{n} ; k\right)$ such that $c$ is the equidiameter element of $\mathscr{C}_{n}(k ; G)$ with the maximal radius subject to the condition $\phi(c)=b$. By definition, $\phi \circ \psi=1$. We define the $G \times \widetilde{S}_{k}$-homotopy $h: \psi \circ \phi \simeq_{G \times \mathfrak{\Im}_{k}} 1$ as follows. Let $c \in \mathscr{C}_{n}(k ; G)$ be described as above and $d$ be the radius of $\psi \circ \phi(c)$. Let $d^{\prime}$ be the radius of the maximal equidiameter element $c^{\prime}$ subject to the condition $\operatorname{Im} c^{\prime} \subset \operatorname{Im} c$. Then define

$$
h(c, t)=\left(c_{11}(t) \times \cdots \times c_{1 n}(t), \cdots, c_{k 1}(t) \times \cdots \times c_{k n}(t)\right), 0 \leqq t \leqq 1,
$$

where

$$
c_{i j}(t)(v)= \begin{cases}x_{i j}+\left((1-2 t) \varepsilon_{i j}+2 t d^{\prime}\right) v, & 0 \leqq t \leqq \frac{1}{2}, \\ x_{i j}+\left((2-2 t) d^{\prime}+(2 t-1) d\right) v, & \frac{1}{2} \leqq t \leqq 1 .\end{cases}
$$

This gives the $G \times \widetilde{S}_{k}$-homotopy from 1 to $\psi \circ \phi$.

There is an inclusion $i_{n}: \mathscr{C}_{n}(k ; G) \rightarrow \mathscr{C}_{n+1}(k ; G)$ defined by $i_{n}\left(\left(c_{1}, \cdots, c_{k}\right)\right)$ $\left(c_{1} \times 1, \cdots, c_{k} \times 1\right)$, which is a $G \times \widetilde{S}_{k}$-map. We define $\mathscr{C}_{\infty}(k ; G)=\operatorname{colim}_{n} \mathscr{C}_{n}$ $(k ; G) . \quad \mathscr{C}_{\infty}(k ; G)$ receives the $G \times \widetilde{S}_{k}$-action which is $\mathfrak{S}_{k}$-free. Let $i_{n}^{\prime}$; $F\left(\left(\check{D}_{\rho} \rho\right)^{n} ; k\right) \rightarrow F\left(\left(\check{D}_{\rho}\right)^{n+1} ; k\right)$ be the inclusion map induced from the map $\left(\check{D}_{\rho}\right)^{n}$ $\rightarrow\left(\dot{D}_{\rho} \rho\right)^{n+1},\left(v_{1}, \cdots, v_{n}\right) \rightarrow\left(v_{1}, \cdots, v_{n}, 0\right)$. Let $\phi_{n}: \mathscr{C}_{\infty}(k ; G) \rightarrow F\left(\left(\dot{D}_{\rho}\right)^{n} ; k\right)$ be the $G \times \Im_{k}$-homotopy equivalence defined in the proof of (1.2). As the following diagram

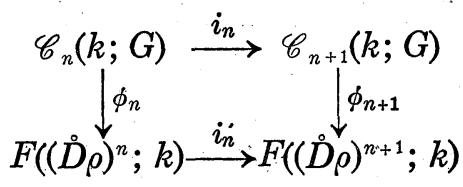

is commutative and $F\left(\left(\check{D}_{\rho}\right)^{n} ; k\right)$ has the $G \times \widetilde{S}_{k}$-homotopy type of a $G \times \widetilde{S}_{k}$ complex, $\mathscr{C}_{\infty}(k ; G)$ has the $G \times \mathscr{S}_{k}$-homotopy type of a $G \times \widetilde{S}_{k}$-complex and $\operatorname{colim}_{n} \phi_{n}: \mathscr{C}_{\infty}(k ; G) \rightarrow \operatorname{colim}_{n} F\left(\left(\stackrel{D}{ } \rho_{\rho}\right)^{n} ; k\right)$ is a $G \times \mathfrak{S}_{k}$-homotopy equivalence 
(Waner [15], Lemma 4.8).

Propositron 1.3. Let $\tilde{H}$ be a subgroup of $G \times \mathscr{S}_{k}$. The fixed-point set $\mathscr{C}_{\infty}(k ; G)^{\tilde{H}}$ is contractible if $\tilde{H}=\left\{(h, \tau(h)) \in G \times \widetilde{S}_{k} \mid h \in H\right\}$ for a homomorphism $\tau: H \rightarrow \widetilde{S}_{k}$, where $H$ is a subgroup of $G$, and is empty otherwise.

Proof. Assume $\mathscr{C}_{\infty}(k ; G)^{\tilde{H}}$ is not empty. Put $H=p r_{1}(\tilde{H}), p r_{1} ; G \times \widetilde{S}_{k} \rightarrow$ G. The map $\tau: H \rightarrow \mathbb{S}_{k}$ defined by $(h, \tau(h)) \in \tilde{H}, h \in H$, is a well defined homomorphism because $\mathscr{C}_{\infty}(k ; G)$ is $\Im_{k}$-free. $F(C[G] ; k)=\left(C[G]^{n}\right)^{k}-\Delta$, where $\Delta=$ $\left\{\left(x_{1}, \cdots, x_{k}\right) \in\left(C[G]^{n}\right)^{k} \mid x_{i}=x_{j}\right.$ for some $\left.i \neq j\right\}$, and therefore $F\left(C[G]^{n} ; k\right)^{\tilde{H}}=$ $\left(\left(C[G]^{n}\right)^{k}\right)^{\tilde{H}}-\Delta$. Let $G=H g_{1} \amalg \cdots \amalg H g_{r}$ be the left coset decomposition. We denote a point of $\left(C[G]^{n}\right)^{k}$ by $\left(\sum_{g \in G} a_{g, m, l} g\right)_{1 \leqq m \leqq n, 1 \leqq l \leqq k}$, where $a_{g, m, l} \in C$. Then

$$
\begin{aligned}
& \left(\sum_{g \in G} a_{g, m, l} g\right)_{m, l} \in\left(\left(C[G]^{n}\right)^{k}\right)^{\tilde{H}} \\
\Longleftrightarrow & \left(\sum_{g \in G} a_{g, m, \tau(h)-1(l)} h g\right)_{m, l}=\left(\sum_{g \in G} a_{g, m, l} g\right)_{m, l} \\
\Longleftrightarrow & a_{h-1 g_{i}, m, \tau(h)-1(l)}=a_{g_{i}, m, l} \quad \text { for all } h \in H, \\
& 1 \leqq i \leqq|G| H \mid, 1 \leqq m \leqq n \text { and } \quad 1 \leqq l \leqq k
\end{aligned}
$$

Therefore the restriction of the projection map pr: $\left(C[G]^{n}\right)^{k} \rightarrow C^{|G / H| n k}$, $\operatorname{pr}\left(\left(\sum_{g \in G} a_{g, m, l} g\right)_{m, l}\right)=\left(a_{g_{i}, m, l}\right)_{i, m, l}$, to $\left(\left(C[G]^{n}\right)^{k}\right)^{\tilde{H}}$ is a homeomorphism. Then $\left(\left(C[G]^{n}\right)^{k}\right)^{\tilde{H}}-\Delta \approx C^{|G / H| n k}-p r\left(\left(\left(C[G]^{n}\right)^{k}\right)^{\tilde{H}} \cap \Delta\right)$ and $\Delta^{\prime}=\operatorname{pr}\left(\left(\left(C[G]^{n}\right)^{k}\right)^{\tilde{H}} \cap \Delta\right) \subset \operatorname{pr}(\Delta)$. Let $\left(\Sigma_{g \in G} a_{g, m, l} g\right)_{m, l} \in\left(\left(C[G]^{n}\right)^{k}\right)^{\tilde{H}}$. Then $\left(\Sigma_{g \in G} a_{g, m, l} g\right)_{m, l} \in\left(\left(C[G]^{n}\right)^{k}\right)^{\tilde{H}} \cap \Delta$ iff there are $l_{1}$ and $l_{2}$ such that $l_{1} \neq l_{2}$ and $a_{g, m, l_{1}}=a_{g, m, l_{2}}$ for all $g \in G$ and $1 \leqq m \leqq$ $n$, which is equivalent to saying that there are $l_{1}$ and $l_{2}$ such that $l_{1} \neq l_{2}$ and $a_{g_{i}, m, \tau(h)-1\left(l_{1}\right)}=a_{g_{i}, m, \tau(h)-1\left(l_{2}\right)}$ for all $h \in H, 1 \leqq i \leqq|G / H|$ and $1 \leqq m \leqq n$. Therefore $\Delta^{\prime}$ is a union of linear subspaces contained in $\operatorname{pr}(\Delta)$. Take a triangulation of $C^{|G / H| n k}$ containing $\Delta^{\prime}$ as a subcomplex. Then $\operatorname{dim} C^{|G / H| n k}-\operatorname{dim} \Delta^{\prime} \geqq$ $\operatorname{dim} C^{|G / H| n k}-\operatorname{dim} \operatorname{pr}(\Delta)=2|G / H| n$. Therefore by the usual argument of celluler approximations with respect to the dual cell complex of this polyhedron, we see that $C^{|G / H| n k}-\Delta^{\prime}$ is $(2|G / H| n-2)$-connected. Therefore

$$
\operatorname{colim}_{n} F\left(C[G]^{n} ; k\right)^{\tilde{H}} \text { and } \mathscr{C}_{\infty}(k ; G)^{\tilde{H}}
$$

are contractible.

If $\tilde{H}=\left\{(h, \tau(h)) \in G \times \widetilde{S}_{k} \mid h \in H\right\}$ for a homomorphism $\tau: H \rightarrow \mathfrak{S}_{k}, H \leqq G$, it is easily checked that $\mathscr{C}_{\infty}(k ; G)^{\tilde{H}}$ is non empty by (1.4).

Let $X$ be a based $G$-space. Define the map

$$
\theta_{n, k}^{\prime}: \mathscr{C}_{n}(k ; G) \times\left(\Omega^{n \rho} X\right)^{k} \rightarrow \Omega^{n \rho} X
$$

by

$$
\theta_{n, k}^{\prime}\left(c, f_{1}, \cdots, f_{k}\right)(v)=\left\{\begin{array}{c}
f_{i} \circ c_{i}^{-1}(v), v \in \operatorname{Im} c_{i}, \\
*, v \notin \operatorname{Im}^{*} c_{i},
\end{array}\right.
$$


where $c=\left(c_{1}, \cdots, c_{k}\right) \in \mathscr{C}_{n}(k ; G), f_{i} \in \Omega^{n_{\rho}} X$ and $v \in\left(\dot{D}_{\rho}\right)^{n} . \quad \theta_{n, k}^{\prime}$ is $G \times \mathfrak{S}_{k}$-equivariant and induces the $G$-map

$$
\theta_{n, k}: \mathscr{C}_{n}(k ; G)_{+} \wedge \varsigma_{\Theta_{k}}\left(\Omega^{n \rho} X\right)^{k} \rightarrow \Omega^{n \rho} X .
$$

Let $Y_{0}$ be an infinite loop $G$-space. There is a sequence $\left\{Y_{i}\right\}_{i \geqq 0}$ of $G$ spaces such that $Y_{n} \simeq{ }_{G} \Omega^{\rho} Y_{n+1}$. Then $\theta_{n, k}$ induces the $G$-map

$$
\theta_{\infty, k}: \mathscr{C}_{\infty}(k ; G)_{+} \bigwedge_{\varsigma_{k}}\left(Y_{0}\right)^{k} \rightarrow Y_{0} .
$$

\section{§ 2. Equivariant transfers and wreath operations}

We call $p: X \rightarrow Y$ a $k$-fold $G$-covering if $X$ and $Y$ are $G$-spaces and $p$ is a $k$-fold covering $G$-map. Let $Z=\left\{z:[k] \rightarrow X \mid z([k])=p^{-1}(y)\right.$ for some $\left.y \in Y\right\},[k]$ $=\{1, \cdots, k\}$, and $\tilde{p}: Z \rightarrow Y$ be $\tilde{p}(z)=y$ if $z([k])=p^{-1}(y)$. $\quad G$ acts on $Z$ by $(g z)(i)$ $=g(z(i))$. Then $\tilde{p}$ is a principal $\left(G, \widetilde{S}_{k}\right)$-bundle in the sense of tom Dieck [14]. $p$ is the $G$-bundle with fiber $[k]$ associated with $\tilde{p}$. Let $\Gamma$ be a subgroup of $\widetilde{S}_{k}$ and $\operatorname{Cov}_{G}^{T}$ be the category whose objects are $k$-fold $G$-coverings $p:(X, A)$ $\rightarrow(Y, B), A=p^{-1}(B)$, with structure group $\Gamma$ such that $(Y, B)$ have the $G$ homotopy type of pairs of $G$-complexes and morphisms are pullback diagrams

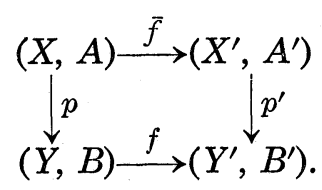

Let $\mathscr{T}_{G *}$ be the category of based $G$-spaces and $G$-homotopy classes of based $G$-maps as morphisms and $\mathscr{W}_{G *}$ be the full subcategory of $\mathscr{T}_{G *}$ consisting of based $G$-spaces having the $G$-homotopy types of based $G$-complexes. Let $Q_{G}$ be the functor from $\mathscr{W}_{G *}$ to $\mathscr{T}_{G *}$ defined by $Q_{G}(X)=\operatorname{colim}_{n} \Omega^{n \rho} \sum^{n \rho} X$, the colimit of $\Omega^{n \rho}(\operatorname{adj} 1): \Omega^{n \rho} \Sigma^{{ }^{n} \rho} X \rightarrow \Omega^{(n+1) \rho} \Sigma^{(n+1) \rho} X$, where adj 1 is an adjoint map of $1_{\Sigma^{(n+1) \rho_{X}}}$. Let $F_{1}: \operatorname{Cov}_{G}^{T} \rightarrow \mathscr{T}_{G *}$ be the functor defined by $F_{1}(p)=Y \mid B$ for $p:(X, A) \rightarrow(Y, B) \in \operatorname{Cov}_{G}^{\Gamma}$ and $F_{1}((f, \bar{f}))=f: Y \mid B \rightarrow Y^{\prime} / B^{\prime}$ for $(f, \bar{f})$ of $(2.1)$, and $F_{2}: \operatorname{Cov}_{G}^{\Gamma} \rightarrow \mathscr{T}_{G *}$ be the functor defined by $F_{2}(p)=Q_{G}(X \mid A)$ for $p \in \operatorname{Cov}_{G}^{T}$ and $F_{2}((f, \bar{f}))=Q_{G}(\bar{f}): Q_{G}(X / A) \rightarrow Q_{G}\left(X^{\prime} \mid A^{\prime}\right)$.

Definition 2.2. A generalized equivariant transfer is a natural transformation $t: F_{1} \rightarrow F_{2}$. An equivariant transfer $t$ is a generalized equivariant transfer satisfying the following axioms (T1) and (T2).

(T1) The following diagram

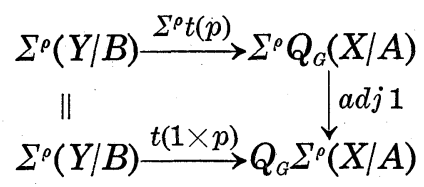


is commutative in $\mathscr{T}_{G *}$, where $p:(X, A) \rightarrow(Y, B) \in \operatorname{Cov}_{G}^{\Gamma}, 1 \times p:(D \rho \times Y, D \rho \times$ $A \cup S \rho \times X) \rightarrow(D \rho \times Y, D \rho \times B \cup S \rho \times Y) \in \operatorname{Cov}_{G}^{T}$ and $a d j 1$ is an adjoint map of 1: $Q_{G}(X \mid A) \approx{ }_{a} Q^{\circ} Q_{G} \Sigma^{\rho}(X \mid A)$.

(T2) Let $p:(X, A) \rightarrow(Y, B) \in \operatorname{Cov}_{G}^{\Gamma},(X, A)=\left(X^{\prime}, A^{\prime}\right) \amalg(Y, B)$ and $\left.p\right|_{(Y, B)}=$ $1_{(Y, B)}$. Then the composition

$$
Y \mid B \stackrel{t(p)}{\longrightarrow} Q_{\theta}(X / A)=Q_{G}\left(X^{\prime} \mid A^{\prime} \vee Y / B\right) \stackrel{c}{\longrightarrow} Q_{G}(Y / B)
$$

is equal to the canonical inclusion map $i: Y \mid B \rightarrow Q_{G}(Y \mid B)$ in $\mathscr{T}_{a *}$, where $c$ collapses $X^{\prime} \mid A^{\prime}$.

We will prove the following theorem in this and the next sections.

THEOREM 2.3. The equivariant transfer on Cov $\operatorname{Cox}_{G}^{T}$ exists uniquely.

Let $p:(X, A) \rightarrow(Y, B) \in \operatorname{Cov}_{F}^{T}$ and $\tilde{p}:(Z, C) \rightarrow(Y, B)$ be the associated principal $(G, \Gamma)$-bundle. Let $\tilde{H}$ be a subgroup of $G \times \Gamma$. If $Z^{\tilde{H}}=\phi$ then $\tilde{H}=$ $\{(h, \tau(h)) \mid h \in H\}$ for a homomorphism $\tau: H \rightarrow \Gamma$, where $H$ is a subgroup of $G$, and $\mathscr{C}_{\infty}(k ; G)^{\mathscr{H}}$ is contractible by Proposition 1.3. Therefore there is a unique $G \times \Gamma$-map $d: Z \rightarrow \mathscr{C}_{\infty}(k ; G)$ up to $G \times \Gamma$-homotopy (Bredon [4], Chapter II).

In a way parallel to [13], p. 98, we consider the following diagram

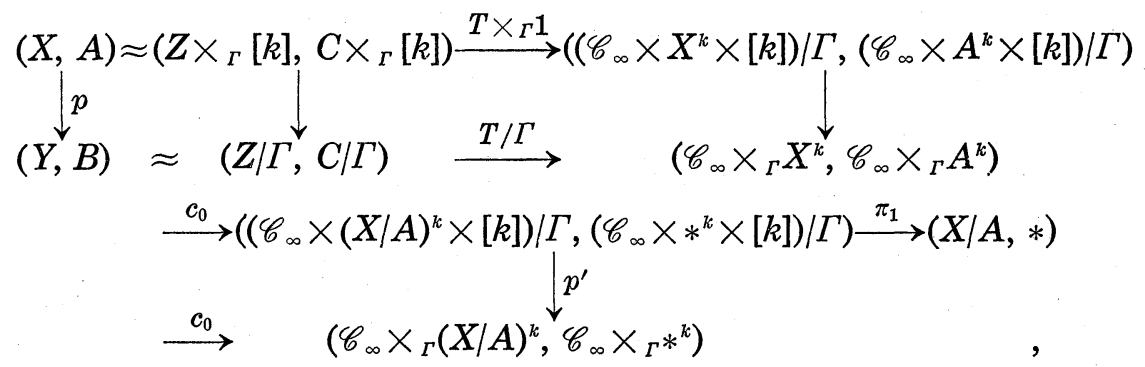

where $\mathscr{C}_{\infty}=\mathscr{C}_{\infty}(k ; G), T=\left(d, f_{1}, \cdots, f_{k}\right): Z \rightarrow \mathscr{C}_{\infty} \times X^{k}, f_{i}(z)=z(i), c_{0}$ is the map induced by $X \rightarrow X / A$ and $\pi_{1}\left(\left(c, x_{1}, \cdots, x_{k}, i\right)\right)=x_{i}$. Each squares are pullback diagrams. Let $x \in X$ correspond to $[z, i] \in Z \times_{\Gamma}[k]$ by the canonical homeomorphism $X \approx Z \times{ }_{\Gamma}[k]$. Then

$$
\pi_{1} \circ c_{0} \circ\left(T \times{ }_{T} 1\right)([z, i])=\pi_{1} \circ c_{0}([d(z), z(1), \cdots, z(k), i])=z(i)=x .
$$

Therefore $\pi_{1} \circ c_{0} \circ\left(T \times{ }_{\Gamma} 1\right):(X, A) \rightarrow(X / A, *)$ is the canonical collapsing map.

Let $F_{3}: \mathscr{W}_{G^{*}} \rightarrow \mathscr{T}_{G^{*}}$ be the functor defined by $F_{3}(X)=\mathscr{C}_{\infty}(k ; G)_{+} \bigwedge_{\Gamma} X^{k}$ for $X \in \mathscr{W}_{G *}$ and $F_{3}([f])=\left[1_{+} \bigwedge_{\Gamma} f\right]$ for $[f] \in\left[X, X^{\prime}\right]^{G}$.

DefinITION 2.5. An equivariant wreath operation is a natural transformation $w: F_{3} \rightarrow Q_{G}$.

Let $w$ be an equivariant wreath operation. We construct a generalized 
equivariant transfer $t$ from $w$ as follows. For $p:(X, A) \rightarrow(Y, B) \in \operatorname{Cov}_{G}^{T}, c_{0} \circ T \mid \Gamma$ in (2.4) induces a $G$-map $Y \mid B \rightarrow \mathscr{C}_{\infty}(k ; G)_{+} \bigwedge_{T}(X / A)^{k}$. We define $t(p)$ to be the following composition.

$$
t(p): Y \mid B \rightarrow \mathscr{C}_{\infty}(k ; G)_{+} \bigwedge_{\Gamma}(X / A)^{k} \stackrel{w(X / A)}{\longrightarrow} Q_{G}(X / A) .
$$

For a pullback diagram (2.1), let $\tilde{p}:(Z, C) \rightarrow(Y, B)$ and $\tilde{p}^{\prime}:\left(Z^{\prime}, C^{\prime}\right) \rightarrow\left(Y^{\prime}, B^{\prime}\right)$ be the principal $\Gamma$-bundles associated with $p$ and $p^{\prime}$. The following diagram is commutative.

$$
\begin{aligned}
& (Z, C) \stackrel{\tilde{f}}{\longrightarrow}\left(Z^{\prime}, C^{\prime}\right) \\
& \underset{\sim}{\mid \tilde{p}} \underset{\left(\tilde{p}^{\prime}, B\right) \stackrel{f}{\longrightarrow}}{\longrightarrow}\left(Y^{\prime}, B^{\prime}\right),
\end{aligned}
$$

Let $d^{\prime}: Z^{\prime} \rightarrow \mathscr{C}_{\infty}(k ; G)$ be a $G \times \Gamma$-map and put $d=d^{\prime} \circ f$. Then $d: Z \rightarrow \mathscr{C}_{\infty}(k ; G)$ is a $G \times \Gamma$-map and

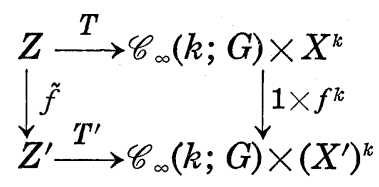

is commutative, where

$$
T(z)=(d(z), z(1), \cdots, z(k)) \quad \text { and } \quad T^{\prime}\left(z^{\prime}\right)=\left(d^{\prime}\left(z^{\prime}\right), z^{\prime}(1), \cdots, z^{\prime}(k)\right) .
$$

Therefore the left square of the following diagram is commutative.

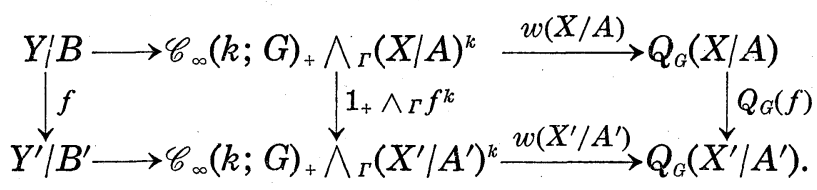

The right square is commutative by the naturality of $w$. Therefore $t$ defined by (2.6) is a generalized equivariant transfer.

Let $t$ be a generalized equivariant transfer. Then we construct an equivariant wreath operation $w$ from this $t$ as follows. Lat $p^{\prime}:\left(\left(\mathscr{C}_{\infty} \times X^{k} \times[k]\right) / \Gamma\right.$, $\left.\left(\mathscr{C}_{\infty} \times *^{k} \times[k]\right) / \Gamma\right) \rightarrow\left(\mathscr{C}_{\infty} \times{ }_{\Gamma} X^{k}, \mathscr{C}_{\infty} \times{ }_{\Gamma} *^{k}\right), \mathscr{C}_{\infty}=\mathscr{C}_{\infty}(k ; G)$, be the $k$-fold $G$-covering defined by the projection. For a based $G$-space $X$, define $w(X)$ to be the following composition.

$$
w(X): \mathscr{C}_{\infty}(k ; G)_{+} \bigwedge_{\Gamma} X^{k} \stackrel{t\left(p^{\prime}\right)}{\longrightarrow} Q_{G}\left(\left(\mathscr{C}_{\infty+} \wedge X^{k} \wedge[k]_{+}\right) / \Gamma\right) \stackrel{Q_{G}\left(\pi_{1}\right)}{\longrightarrow} Q_{G}(X) .
$$

This $w(X)$ is well defined as a $G$-homotopy class. For a $G$-map $f: X \rightarrow X^{\prime}$, the diagram 


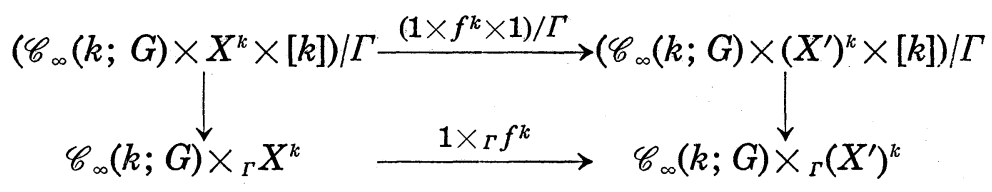

is a pullback diagram. Therefore $w$ is an equivariant wreath operation because of the naturality of $t$.

By an argument parallel to [13], Chapter VII, we get the following lemmas.

LEMma 2.8. (2.6) and (2.7) give $a$ one to one correspondence between equivariant wreath operations and generalized equivariant transfers.

LEMma 2.9. A generalized equivariant transfer $t$ satisfies the axiom (T1) if and only if the associated equivariant wreath operation $w$ satisfies the following axiom:

(W1) The diagram

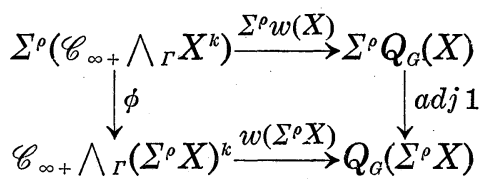

is commutative in $\mathscr{T}_{G *}$, where $\left(v \wedge\left[c \wedge\left(x_{1}, \cdots, x_{k}\right)\right]\right)=\left[c \wedge\left(v \wedge x_{1}, \cdots, v \wedge x_{k}\right)\right]$.

LEMma 2.10. A generalized equivariant transfer $t$ satisfies (T2) iff the associated equivariant wreath operation $w$ satisfies the following axiom:

(W2) The diagram

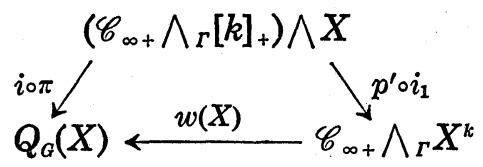

is commutative in $\mathscr{T}_{G *}$, where

$$
i_{1}:\left(\left(\mathscr{C}_{\infty} \times_{\Gamma}[k]\right) \times X,\left(\mathscr{C}_{\infty} \times_{\Gamma}[k]\right) \times *\right) \rightarrow\left(\left(\mathscr{C}_{\infty} \times X^{k} \times[k]\right) / \Gamma,\left(\mathscr{C}_{\infty} \times *^{k} \times[k]\right) / \Gamma\right)
$$

is given by $i_{1}(([c, i], x))=[c, *, \cdots, *, x, *, \cdots, *, i]$ where $x$ is in the $i$-th place, $\pi:\left(\left(\mathscr{C}_{\infty} \times_{\Gamma}[k]\right) \times X,\left(\mathscr{C}_{\infty} \times{ }_{\Gamma}[k]\right) \times *\right) \rightarrow(X, *)$ is the projection to $X$ and $i: X \rightarrow$ $Q_{G}(X)$ is the canonical inclusion.

\section{§ 3. Proof of Theorem 2.3}

Define

$$
\phi: \Sigma^{\rho}\left(\mathscr{C}_{\infty+} \bigwedge_{\Gamma}\left(\Sigma^{n \rho} X\right)^{k}\right) \rightarrow \mathscr{C}_{\infty+} \bigwedge_{\Gamma}\left(\Sigma^{(n+1) \rho} X\right)^{k}
$$


by

$$
\phi\left(v \wedge\left[c \wedge\left(v_{1} \wedge x_{1}, \cdots, v_{k} \wedge x_{k}\right)\right]\right)=\left[c \wedge\left(v \wedge v_{1} \wedge x_{1}, \cdots, v \wedge v_{k} \wedge x_{k}\right)\right] .
$$

Let Tel $\Omega^{n \rho}\left(\mathscr{C}_{\infty+} \bigwedge_{\Gamma}\left(\sum^{n \rho} X\right)^{k}\right)$ be the telescope of the sequence $\mathscr{C}_{\infty+} \bigwedge_{\Gamma} X^{k} \rightarrow \cdots \rightarrow \Omega^{n \rho}\left(\mathscr{C}_{\infty+} \bigwedge_{\Gamma}\left(\Sigma^{n \rho} X\right)^{k}\right) \stackrel{\Omega^{n \rho} a d j \phi}{\longrightarrow} \Omega^{(n+1) \rho}\left(\mathscr{C}_{\infty+} \bigwedge_{\Gamma}\left(\Sigma^{(n+1) \rho} X\right)^{k}\right) \rightarrow \cdots$

Define

$$
\psi: \Sigma^{\rho}\left(\left(\mathscr{C}_{\infty+} \bigwedge_{\Gamma}[k]_{+}\right) \wedge \Sigma^{n \rho} X\right) \rightarrow\left(\mathscr{C}_{\infty+} \bigwedge_{\Gamma}[k]_{+}\right) \wedge \Sigma^{(n+1) \rho} X
$$

by

$$
\psi\left(v \wedge[c \wedge i] \wedge v^{\prime} \wedge x\right)=[c \wedge i] \wedge v \wedge v^{\prime} \wedge x
$$

Let Tel $\Omega^{n \rho}\left(\left(\mathscr{C}_{\infty+} \bigwedge_{\Gamma}[k]_{+}\right) \wedge \Sigma^{n \rho} X\right)$ be the telescope of the sequence

$$
\begin{aligned}
\left(\mathscr{C}_{\infty+} \bigwedge_{\Gamma}[k]_{+}\right) & \wedge X \rightarrow \cdots \rightarrow \Omega^{n \rho}\left(\left(\mathscr{C}_{\infty+} \bigwedge_{\Gamma}[k]_{+}\right) \wedge \Sigma^{n \rho} X\right) \\
& \stackrel{\Omega^{n \rho} a d j \psi}{\longrightarrow} \Omega^{(n+1) \rho}\left(\left(\mathscr{C}_{\infty+} \bigwedge_{\Gamma}[k]_{+}\right) \wedge \Sigma^{(n+1) \rho} X\right) \rightarrow \cdots .
\end{aligned}
$$

The following diagram is commutative.

$$
\begin{gathered}
\Omega^{n \rho}\left(\left(\mathscr{C}_{\infty+} \bigwedge_{\Gamma}[k]_{+}\right) \wedge \Sigma^{n \rho} X\right) \stackrel{\stackrel{\Omega^{n \rho}\left(p^{\prime} \circ i_{1}\right)}{\longrightarrow}}{\longrightarrow} \Omega^{n \rho}\left(\mathscr{C}_{\infty+} \bigwedge_{\Gamma}\left(\Sigma^{n \rho} X\right)^{k}\right) \\
\Omega_{\Omega^{(n+1) \rho}(\operatorname{adj} \psi)}\left(\left(\mathscr{C}_{\infty+} \bigwedge_{\Gamma}[k]_{+}\right) \wedge \Sigma^{(n+1) \rho} X\right) \stackrel{\Omega^{(n+1) \rho}\left(p^{\prime} \circ i_{1}\right)}{\longrightarrow} \Omega^{(n+1) \rho}\left(\mathscr{C}_{\infty+} \bigwedge_{\Gamma}\left(\Sigma^{(n+1) \rho} X\right)^{k}\right),
\end{gathered}
$$

which induces the $G$-map

$$
\widetilde{p^{\prime} \circ i_{1}}: \operatorname{Tel} \Omega^{n \rho}\left(\left(\mathscr{C}_{\infty+} \bigwedge_{\Gamma}[k]_{+}\right) \wedge \Sigma^{n \rho} X\right) \longrightarrow \operatorname{Tel} \Omega^{n_{\rho}}\left(\mathscr{C}_{\infty+} \bigwedge_{\Gamma}\left(\sum^{n \rho} X\right)^{k}\right) .
$$

A $G$-map

$$
\widetilde{i \circ \pi}: \operatorname{Tel} \Omega^{n \rho}\left(\left(\mathscr{C}_{\infty+} \bigwedge_{\Gamma}[k]_{+}\right) \wedge \Sigma^{n \rho} X\right) \longrightarrow Q_{G}(X)
$$

is defined from the following commutative diagram

$$
\begin{gathered}
\Omega^{n \rho}\left(\left(\mathscr{C}_{\infty+} \bigwedge_{\Gamma}[k]_{+}\right) \wedge \Sigma^{n \rho} X\right) \stackrel{\stackrel{\Omega^{n \rho}(i \circ \pi)}{\longrightarrow} \Omega^{n \rho} Q_{G}\left(\Sigma^{n \rho} X\right)}{\left.\right|^{n \rho} \operatorname{adj} \psi} \\
\Omega^{(n+1) \rho}\left(\left(\mathscr{C}_{\infty+} \bigwedge_{\Gamma}[k]_{+}\right) \wedge \Sigma^{(n+1) \rho} X\right) \stackrel{\Omega^{(n+1) \rho}(i \circ \pi)}{\longrightarrow} \Omega^{(n+1) \rho} Q_{G}\left(\Sigma^{(n+1) \rho} X\right) .
\end{gathered}
$$

Definition 3.1. An equivariant stable wreath operation $\tilde{w}$ is a natural transformation from the functor Tel $\Omega^{n \rho}\left(\mathscr{C}_{\infty+} \bigwedge_{\Gamma}\left(\Sigma^{n_{\rho}}(\quad)\right)^{k}\right)$ to $Q_{G}$ on $\mathscr{W}_{G *}$ such that the following diagram

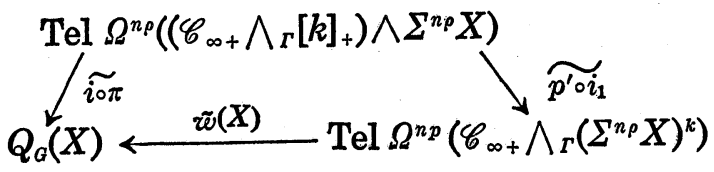


is commutative in $\mathscr{T}_{G *}$.

Let $w$ be an equivariant wreath operation satisfying (W1) and (W2). We construct an equivariant stable wreath operation $\tilde{w}$ from $w$. Though $w(X)$ is defined as a $G$-homotopy class, we denote a representative $G$-map also by the same notation with an abuse of notations. We will show later that the $G$-homotopy class $\tilde{w}(X)$ is independent of choice of representatives of $w(X)$.

Consider the diagram

$$
\begin{gathered}
\Omega^{n \rho}\left(\mathscr{C}_{\infty+} \bigwedge_{\Gamma}\left(\sum^{n \rho} X\right)^{k}\right) \stackrel{\Omega^{n \rho} w\left(\Sigma^{n \rho} X\right)}{\longrightarrow} \Omega^{n p} Q_{G}\left(\sum^{n \rho} X\right) \\
\Omega^{(n+1) \rho}\left(\mathscr { C } _ { \infty + } \bigwedge _ { \Gamma } \left(\Sigma^{n \rho} a d j \phi\right.\right. \\
\left.\left.\Sigma^{(n+1) \rho} X\right)^{k}\right) \stackrel{\Omega^{(n+1) \rho} w\left(\Sigma^{(n+1) \rho} X\right)}{\longrightarrow} \Omega^{(n+1) \rho} Q_{G}\left(\Sigma^{(n+1) \rho} X\right) .
\end{gathered}
$$

By (W1), there is a $G$-homotopy $H^{(n) \prime}: \Sigma^{\rho}\left(\mathscr{C}_{\infty+} \bigwedge_{\Gamma}\left(\Sigma^{n \rho} X\right)^{k}\right) \wedge I_{+} \rightarrow Q_{G}\left(\Sigma^{(n+1) \rho} X\right)$, $I=[0,1]$, from $(\operatorname{adj} 1) \circ \Sigma^{\rho} w\left(\Sigma^{n \rho} X\right)$ to $w\left(\Sigma^{(n+1) \rho} X\right)$. Then a $G$-homotopy $H_{t}^{(n)}$, $0 \leqq t \leqq 1$, from $\Omega^{n \rho} w\left(\Sigma^{n \rho} X\right)$ to $\Omega^{(n+1) \rho} w\left(\Sigma^{(n+1) \rho} X\right) \circ\left(\Omega^{n \rho} a d j \phi\right)$ is defined by $H_{t}^{(n)}$ $=\Omega^{n \rho}\left(\operatorname{adj} H_{t}^{(n) \prime}\right)$, where $H_{t}^{(n) \prime}(y)=H^{(n) \prime}(y \wedge t)$. Define

$$
\tilde{w}(X): \operatorname{Tel} \Omega^{n \rho}\left(\mathscr{C}_{\infty+} \bigwedge_{\Gamma}\left(\Sigma^{n \rho} X\right)^{k}\right) \longrightarrow Q_{G}(X)
$$

by

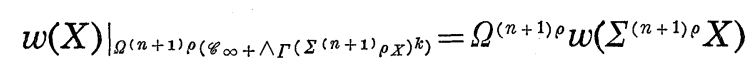

and

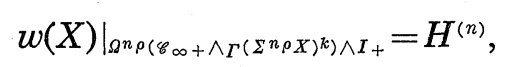

where $H^{(n)}(y \wedge t)=H_{t}^{(n)}(y)$. We show that (3.2) is $G$-homotopy commutative for $\tilde{w}(X)$ defined in this way and prove in (3.5) that $\widetilde{p^{\prime} \circ i_{1}}$ is a $G$-homotopy equivalence. Then $\tilde{w}(X)$ is $G$-homotopic to $(\widetilde{i \circ \pi}) \circ\left(\widetilde{p^{\prime} \circ i_{1}}\right)^{-1}$, where $\left(\widetilde{p^{\prime} \circ i_{1}}\right)^{-1}$ is a $G$-homotopy inverse of $\widetilde{p^{\prime} \circ i_{1}}$ and the $G$-homotopy class of $\tilde{w}(X)$ is independent of representatives of $w(X)$ and of choice of $G$-homotopy $H^{(n) \prime}$.

There is a $G$-homotopy $F^{(0) \prime}:\left(\mathscr{C}_{\infty+} \bigwedge_{\Gamma}[k]_{+}\right) \wedge X \wedge I_{+} \rightarrow Q_{G}(X)$ from $i \circ \pi$ to $w(X) \circ p^{\prime} \circ i_{1}$ by (W2). Assume that we get a $G$-homotopy

$$
F^{(n) \prime}:\left(\mathscr{C}_{\infty+} \bigwedge_{\Gamma}[k]_{+}\right) \wedge \Sigma^{n_{\rho}} X \wedge I_{+} \longrightarrow Q_{G}\left(\Sigma^{n \rho} X\right)
$$

from $i \circ \pi$ to $w\left(\Sigma^{n \rho} X\right) \circ p^{\prime} \circ i_{1}$ in the following diagram

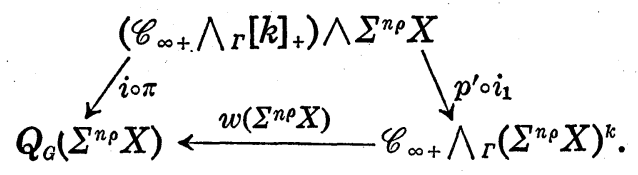


Then we define $F^{(n+1) \prime}$ as follows:

$$
F^{(n+1) \prime}(y \wedge t)= \begin{cases}(a d j 1)\left(\sum^{\rho} F^{(n) \prime}\left(\psi^{-1}(y) \wedge 2 t\right)\right), & 0 \leqq t \leqq 1 / 2, \\ H^{(n) \prime}\left(\Sigma^{\rho}\left(p^{\prime} \circ i_{1}\right) \circ \psi^{-1}(y) \wedge(2 t-1)\right), & 1 / 2 \leqq t \leqq 1\end{cases}
$$

There is a $G$-homotopy $F^{(n)}: \Omega^{n \rho}\left(\left(\mathscr{C}_{\infty_{+}} \bigwedge_{\Gamma}[k]_{+}\right) \wedge \Sigma^{n \rho} X\right) \wedge I_{+} \rightarrow Q_{G}(X)$ defined by $F_{t}^{(n)}=\Omega^{n \rho} F_{t}^{(n) \prime}, 0 \leqq t \leqq 1$. Consider the following diagram

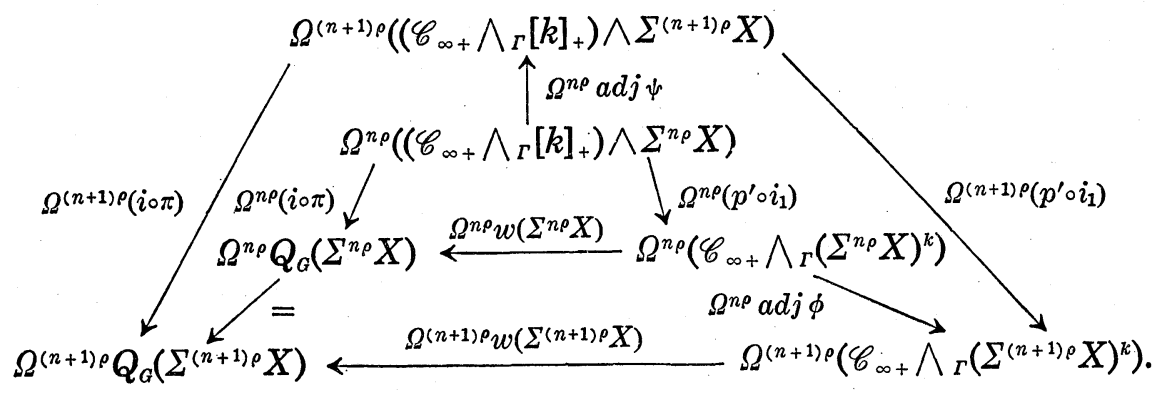

The inner triangle is $G$-homotopy commutative by $F^{(n)}$ and the outer one is so by $F^{(n+1)}$. A $G$-homotopy

$$
K^{(n)}: \Omega^{n \rho}\left(\left(\mathscr{C}_{\infty_{+}+} \wedge_{\Gamma}[k]_{+}\right) \wedge \Sigma^{n \rho} X\right) \wedge I_{+} \wedge I_{+} \longrightarrow Q_{G}(X)
$$

from $F^{(n)}$ to $F^{(n+1)} \circ\left(\Omega^{n \rho} a d j \psi \wedge 1\right)$ is defined as follows. Let

$$
K^{(n) \prime}: \Sigma^{\rho}\left(\left(\mathscr{C}_{\infty+} \bigwedge_{\Gamma}[k]_{+}\right) \wedge \Sigma^{n \rho} X\right) \wedge I_{+} \wedge I_{+} \longrightarrow Q_{G}\left(\Sigma^{(n+1) \rho} X\right)
$$

be a $G$-homotopy from $(\operatorname{adj} 1) \circ \Sigma^{\rho} F^{(n) \prime}$ to $F^{(n+1) \prime} \circ(\psi \wedge 1)$ defined by

$$
K^{(n) \prime}(y \wedge t \wedge u)= \begin{cases}(\operatorname{adj} 1)\left(\Sigma^{\rho} F^{(n) \prime}(y \wedge(2 t /(2-u)))\right), & 0 \leqq t \leqq 1-(u / 2), \\ H^{(n) \prime}\left(\Sigma^{\rho}\left(p^{\prime} \circ i_{1}\right)(y) \wedge(2 t+u-2)\right), & 1-(u / 2) \leqq t \leqq 1 .\end{cases}
$$

Put $K_{t, u}^{(n)}=\Omega^{n \rho} a d j K_{t, u}^{(n) \prime}$ and $K^{(n)}(y \wedge t \wedge u)=K_{t, u}^{(n)}(y)$. Define

$$
K: \operatorname{Tel} \Omega^{n \rho}\left(\left(\mathscr{C}_{\infty+} \bigwedge_{\Gamma}[k]_{+}\right) \wedge \Sigma^{n_{\rho}} X\right) \wedge I_{+} \longrightarrow Q_{G}(X)
$$

by $K([y \wedge u] \wedge t)=K^{(n)}(y \wedge t \wedge u)$ for $[y \wedge u] \wedge t \in \Omega^{n \rho}\left(\left(\mathscr{C}_{\infty+} \wedge_{\Gamma}[k]_{+}\right) \wedge \Sigma^{n \rho} X\right) \wedge I_{+}$ $\wedge I_{+} \subset \mathrm{Tel} \Omega^{n \rho}\left(\left(\mathscr{C}_{\infty+} \wedge_{\Gamma}[k]_{+}\right) \wedge \Sigma^{n_{\rho}} X\right) \wedge I_{+}$. Because of

$$
K^{(n)}(y \wedge t \wedge 1)=F^{(n+1)}\left(\left(\Omega^{n \rho} a d j \psi\right)(y) \wedge t\right)=K^{(n+1)}\left(\left(\Omega^{n \rho} a d j \psi\right)(y) \wedge t \wedge 0\right),
$$

$K$ is well defined on the telescope.

$$
K([y \wedge u] \wedge 0)=K^{(n)}(y \wedge 0 \wedge u)=F^{(n)}(y \wedge 0)=\Omega^{n \rho}(i \circ \pi)(y)
$$

and

$$
K([y \wedge u] \wedge 1)=K^{(n)}(y \wedge 1 \wedge u)=H^{(n)}\left(\Omega^{n \rho}\left(p^{\prime} \circ i_{1}\right)(y) \wedge u\right) .
$$


Therefore $K$ is a $G$-homotopy from $\widetilde{i} \circ \pi$ to $w(X) \circ\left(\widetilde{p^{\prime} \circ i_{1}}\right)$.

Proposition 3.5. $\overparen{p^{\prime} \circ i_{1}}$ is a G-homotopy equivalence.

Proof. It is sufficient to prove that

$\left(\widetilde{p^{\prime} \circ i_{1}}\right)^{H}:\left(\operatorname{Tel} \Omega^{n \rho}\left(\left(\mathscr{C}_{\infty+} \bigwedge_{\Gamma}[k]_{+}\right) \wedge \Sigma^{n \rho} X\right)\right)^{H} \longrightarrow\left(\operatorname{Tel} \Omega^{n_{\rho}}\left(\mathscr{C}_{\infty_{+}} \bigwedge_{\Gamma}\left(\Sigma^{n \rho} X\right)^{k}\right)\right)^{H}$

gives a one to one correspondence of components and homotopy equivalence between each component for any subgroup $H$ of $G$ (Bredon [5], Chapter II, (5.5)).

By the equivariant suspension theorem (Murayama [10], Theorem 2.9; Kakutani-Murayama [8], Theorem 3.6; Namboodiri [11], Theorem 2.3),

$$
\Sigma_{*}^{\rho}:\left[\Sigma^{n \rho},\left(\mathscr{C}_{\infty+} \bigwedge_{\Gamma}[k]_{+}\right) \wedge \Sigma^{n \rho} X\right]^{H} \longrightarrow\left[\Sigma^{(n+1) \rho}, \Sigma^{\rho}\left(\left(\mathscr{C}_{\infty+} \bigwedge_{\Gamma}[k]_{+}\right) \wedge \Sigma^{n \rho} X\right)\right]^{H}
$$

is an isomorphism for large $n$. Then

$\left(\Omega^{n \rho} \operatorname{adj} \psi\right)^{H}:\left(\Omega^{n \rho}\left(\left(\mathscr{C}_{\infty+} \bigwedge_{\Gamma}[k]_{+}\right) \wedge \Sigma^{n \rho} X\right)\right)^{H} \longrightarrow\left(\Omega^{(n+1) \rho}\left(\left(\mathscr{C}_{\infty+} \bigwedge_{\Gamma}[k]_{+}\right) \wedge \Sigma^{(n+1) \rho} X\right)\right)^{H}$ gives a one to one correspondence of components for large $n$. Therefore

$\left(\operatorname{Tel}\left(\Omega^{n \rho}\left(\left(\mathscr{C}_{\infty+} \bigwedge_{\Gamma}[k]_{+}\right) \wedge \Sigma^{n \rho} X\right)\right)^{H}\right)_{[f, t]} \simeq \operatorname{Tel}\left(\left(\Omega^{n \rho}\left(\left(\mathscr{C}_{\infty_{+}} \bigwedge_{\Gamma}[k]_{+}\right) \wedge \Sigma^{n \rho} X\right)\right)_{[f]}^{H}\right)$, where $f \in \Omega^{n \rho}\left(\left(\mathscr{C}_{\infty+} \bigwedge_{\Gamma}[k]_{+}\right) \wedge \sum^{n \rho} X\right), \quad t \in I$, and $\left(\operatorname{Tel}\left(\Omega^{n \rho}\left(\left(\mathscr{C}_{\infty+} \bigwedge_{\Gamma}[k]_{+}\right) \wedge\right.\right.\right.$ $\left.\left.\left.\Sigma^{n \rho} X\right)\right)^{H}\right)_{[f, t]}$ and $\Omega^{n \rho}\left(\left(\mathscr{C}_{\infty+} \bigwedge_{\Gamma}[k]_{+}\right) \wedge \Sigma^{{ }^{n}} X\right)_{[f]}^{H}$ are connected components containing $[f, t]$ and $[f]$. Then

$$
\begin{aligned}
\left(\widetilde{p^{\prime} \circ i_{1}}\right)_{*}^{H}: & {\left[\Sigma^{l}, \operatorname{Tel}\left(\Omega^{n \rho}\left(\left(\mathscr{C}_{\infty+} \bigwedge_{\Gamma}[k]_{+}\right) \wedge \Sigma^{n \rho} X\right)\right)^{H}\right] } \\
\longrightarrow & {\left[\Sigma^{l}, \operatorname{Tel}\left(\Omega^{n \rho}\left(\mathscr{C}_{\infty+} \bigwedge_{\Gamma}\left(\Sigma^{n \rho} X\right)^{k}\right)\right)^{H}\right] }
\end{aligned}
$$

is an isomorphism iff

$$
\begin{aligned}
& \underset{n}{\operatorname{colim}}\left(p^{\prime} \circ i_{1}\right)_{*}^{H}: \operatorname{colim}_{n}\left[\Sigma^{l},\left(\Omega^{n_{\rho}}\left(\left(\mathscr{C}_{\infty+} \bigwedge_{\Gamma}[k]_{+}\right) \wedge \Sigma^{n_{\rho}} X\right)\right)^{H}\right] \\
& \longrightarrow \operatorname{colim}_{n}\left[\Sigma^{l},\left(\Omega^{n \rho}\left(\mathscr{C}_{\infty+} \bigwedge_{\Gamma}\left(\Sigma^{n \rho} X\right)^{k}\right)\right)^{H}\right]
\end{aligned}
$$

is so, which is equivalent to saying that

$$
\begin{gathered}
\underset{n}{\operatorname{colim}\left(p^{\prime} \circ i_{1}\right)_{*}}: \underset{n}{\operatorname{colim}}\left[\Sigma^{n \rho} \wedge \Sigma^{l} \wedge G / H_{+},\left(\mathscr{C}_{\infty+} \bigwedge_{\Gamma}[k]_{+}\right) \wedge \Sigma^{n \rho} X\right]^{G} \\
\longrightarrow \operatorname{colim}_{n}\left[\Sigma^{n \rho} \wedge \Sigma^{l} \wedge G / H_{+}, \mathscr{C}_{\infty+} \bigwedge_{\Gamma}\left(\Sigma^{n \rho} X\right)^{k}\right]^{G}
\end{gathered}
$$

is an isomorphism. We will prove that

$$
\begin{aligned}
\left(p^{\prime} \circ i_{1}\right)_{*}: & {\left[\Sigma^{n \rho} \wedge \Sigma^{l} \wedge G / H_{+},\left(\mathscr{C}_{\infty+} \bigwedge_{\Gamma}[k]_{+}\right) \wedge \Sigma^{n \rho} X\right]^{G} } \\
\longrightarrow & {\left[\Sigma^{n \rho} \wedge \Sigma^{l} \wedge G / H_{+}, \mathscr{C}_{\infty+} \bigwedge_{\Gamma}\left(\Sigma^{n \rho} X\right)^{k}\right]^{G} }
\end{aligned}
$$


is an isomorphism for large $n$. To show this it is sufficient to prove that

$$
\left(p^{\prime} \circ i_{1}\right)^{K}:\left(\left(\mathscr{C}_{\infty+} \bigwedge_{\Gamma}[k]_{+}\right) \wedge \Sigma^{n \rho} X\right)^{K} \rightarrow\left(\mathscr{C}_{\infty+} \bigwedge_{\Gamma}\left(\Sigma^{n_{\rho}} X\right)^{k}\right)^{K}
$$

is a $\left(\operatorname{dim}\left(\Sigma^{n \rho} \wedge \Sigma^{l} \wedge G / H_{+}\right)^{K}+1\right)$-equivalence for all subgroups $K$ of $G$ (Murayama [10], Proposition 2.3). Before proving this we prepare a number of lemmas.

We say $\tau^{\prime} \in \operatorname{Hom}(K, \Gamma)$ is conjugate to $\tau \in \operatorname{Hom}(K, \Gamma)$ if $\tau^{\prime}(a)=\gamma^{-1} \tau(a) \gamma$ for a suitable $\gamma \in \Gamma$ and for all $a \in K$. Then we denote $\tau \sim \tau^{\prime}$. Let $(\tau) \in$ $\operatorname{Hom}(K, \Gamma) / \sim$ be the conjugate class of $\tau$.

LemMa 3.7. Let $X \in \mathscr{W}_{G *}$ and $K$ be a subgroup of $G$.

i ) $\left(\mathscr{C}_{\infty} \times_{\Gamma}\left([k]_{+} \wedge X\right)\right)^{K}=\coprod_{(\tau)}\left(\coprod_{\tau^{\prime} \in(\tau)} \mathscr{C}_{\infty}^{K\left(\tau^{\prime}\right)} \times\left([k]_{+}^{\tau^{\prime}(K)} \wedge X^{K}\right)\right) / \Gamma$,

ii) $\left(\mathscr{C}_{\infty} \times{ }_{\Gamma} X^{k}\right)^{K}=\coprod_{(\tau)}\left(\coprod_{z^{\prime} \in(\tau)} \mathscr{C}_{\infty}^{K\left(\tau^{\prime}\right)} \times\left(X^{k}\right)^{K\left(z^{\prime}\right)}\right) / \Gamma$,

iii) $\left(\mathscr{C}_{\infty} / \Gamma\right)^{K}=\coprod_{(\tau)}\left(\coprod_{\tau^{\prime} \in(\tau)} \mathscr{C}_{\infty}^{K\left(\tau^{\prime}\right)}\right) / \Gamma$,

where $(\tau)$ runs over the conjugate classes $\operatorname{Hom}(K, \Gamma) / \sim$ and $K\left(\tau^{\prime}\right)=\left\{\left(a, \tau^{\prime}(a)\right)\right.$ $\in K \times \Gamma \mid a \in K\}$.

Proof. We will give the proof only for i) as the proofs of ii) and iii) are similar and easier. Let $\pi: \mathscr{C}_{\infty} \times\left([k]_{+} \wedge X\right) \rightarrow \mathscr{C}_{\infty} \times_{\Gamma}\left([k]_{+} \wedge X\right)$ be the projection. It is sufficient to prove the following two facts:

(i) $\pi^{-1}\left(\left(\mathscr{C}_{\infty} \times_{\Gamma}\left([k]_{+} \wedge X\right)\right)^{K}\right)=\coprod_{\tau \in \operatorname{Hom}(K, \Gamma)} \mathscr{C}_{\infty}^{K(\tau)} \times\left([k]_{+}^{\tau(K)} \wedge X^{K}\right)$,

(ii) $\left(\coprod_{\tau^{\prime} \in(\tau)} \mathscr{C}_{\infty}^{K\left(\tau^{\prime}\right)} \times\left([k]_{+}^{z^{\prime}(K)} \wedge X^{K}\right)\right.$ is closed under the $\Gamma$-action.

$\bigcup_{\tau \in \operatorname{Hom}(K, \Gamma)} \mathscr{C}_{\infty}^{K(\tau)} \times\left([k]_{+}^{\tau(K)} \wedge X^{K}\right)=\coprod_{\tau \in \operatorname{Hom}(K, \Gamma)} \mathscr{C}_{\infty}^{K(\tau)} \times\left([k]_{+} \wedge X^{K}\right)$ because $\mathscr{C}_{\infty}$ is $\Gamma$-free. $\quad \pi((c, i \wedge x)) \in\left(\mathscr{C}_{\infty} \times_{\Gamma}\left([k]_{+} \wedge X\right)\right)^{K}$ iff there is a homomorphism $\tau: K$ $\rightarrow \Gamma$ such that $(a c, i \wedge a x)=\left(c \tau(a), \tau(a)^{-1}(i) \wedge x\right)$ for all $a \in K$, which is equivalent to saying that $c \in \mathscr{C}_{\infty}^{K(\tau)}, i \in[k]^{\tau(K)}$ and $x \in X^{K}$. Therefore

$$
\pi^{-1}\left(\left(\mathscr{C}_{\infty} \times_{\Gamma}\left([k]_{+} \wedge X\right)\right)^{K}\right) \subset \coprod_{\tau \in \operatorname{Hom}(K, \Gamma)} \mathscr{C}_{\infty}^{K(\tau)} \times\left([k]_{+}^{\tau(K)} \wedge X^{K}\right) .
$$

The converse inclusion is obvious and (i) is proved. Let $(c, i \wedge x) \in \mathscr{C}_{\infty}^{K(\tau)} \times$ $\left([k]_{+}^{\tau(K)} \wedge X^{K}\right)$ and $\gamma \in \Gamma . \quad(c, i \wedge x) \gamma \in \mathscr{C}_{\infty}^{K\left(\tau^{\prime}\right)} \times\left([k]_{+}^{\tau^{\prime}(K)} \wedge X^{K}\right)$ for a $\tau^{\prime} \in \operatorname{Hom}(K, \Gamma)$ by (i). Then $a(c \gamma)=c \tau(a) \gamma=(c \gamma)\left(\gamma^{-1} \tau(a) \gamma\right)$ and $\tau^{\prime}(a)=\gamma^{-1} \tau(a) \gamma$ for all $a \in K$. Therefore $\tau \sim \tau^{\prime}$ and (ii) is proved.

Let $r=\left|[k]^{\tau(K)}\right|$, the number of the fixed points, for $\tau \in \operatorname{Hom}(K, \Gamma)$ and a subgroup $K$ of $G$. We assume $[k]^{\tau(K)}=\{k-r+1, \cdots, k\}$ for simplicity. Then $\tau=\iota \circ \tilde{\tau}$ for a homomorphism $\tilde{\tau}: K \rightarrow \mathbb{S}_{k-r}$ and $\iota: \mathbb{S}_{k-r} \rightarrow \mathbb{S}_{k}$, where an element $\sigma \in \mathbb{S}_{k-r}$ is considered as a permutation of $k$ letters which leaves the last $r$ letters fixed. Put $\Gamma^{\prime}=\tau(K)$. Let $[k-r] / \Gamma^{\prime}=\left\{\Gamma^{\prime} \cdot i_{1}, \cdots, \Gamma^{\prime} \cdot i_{s}\right\}$ be the orbits and $\Gamma_{l}^{\prime}$ the isotropy group at $i_{l}, 1 \leqq l \leqq s$.

Lemma 3.8. Let $X \in \mathscr{W}_{G *}$. Then

$$
\left(X^{k}\right)^{K(\tau)} \approx X^{\tau-1\left(\Gamma_{1}^{\prime}\right)} \times \cdots \times X^{\tau-1\left(\Gamma_{s}^{\prime}\right)} \times\left(X^{K}\right)^{r} .
$$


Proof. $\left(X^{k}\right)^{K(\tau)} \approx\left(X^{k-r}\right)^{K(z)} \times\left(X^{r}\right)^{K}$ and we prove

$$
\left(X^{k-r}\right)^{K(\tilde{\tau})}=X^{\tau^{-1}\left(\Gamma_{1}^{\prime}\right)} \times \cdots \times X^{\tau^{-1}\left(\Gamma_{s}^{\prime}\right)} .
$$

$K(\tilde{\tau})$ is isomorphic to $K$ by $a \mapsto(a, \tilde{\tau}(a)), a \in K$, and $\operatorname{Ker} \tau$ is considered as a subgroup of $K(\tilde{\tau})$. There are isomorphisms $\alpha: K(\tilde{\tau}) / \operatorname{Ker} \tau \rightarrow \Gamma^{\prime}$ and $\beta: K / \operatorname{Ker} \tau$ $\rightarrow \Gamma^{\prime}$ defined by $\alpha([a, \tilde{\tau}(a)])=\tilde{\tau}(a)$ and $\beta([a])=\tilde{\tau}(a)$. We identify $\Gamma^{\prime}$ with $K(\tilde{\tau}) / \operatorname{Ker} \tau$ by $\alpha$. Then $\left(X^{k-r}\right)^{K(\tilde{z})}=\left(\left(X^{k-r}\right)^{\operatorname{Ker} \tau}\right)^{\Gamma^{\prime}}=\left(\left(X^{\operatorname{Ker} \tau}\right)^{k-r}\right)^{\Gamma^{\prime}}$, where $\Gamma^{\prime}$ acts on $\left(X^{k-r}\right)^{\mathrm{Ker} \tau}$ by $\gamma\left(x_{1}, \cdots, x_{k-r}\right)=\left(\beta^{-1}(\gamma) x_{\gamma^{-1}(1)}, \cdots, \beta^{-1}(\gamma) x_{\gamma^{-1(k-r)}}\right)$ for $\gamma \in \Gamma^{\prime}$. There is a homeomorphism

$$
\left(\left(X^{\mathrm{Ker} \tau}\right)^{k-r}\right)^{\Gamma^{\prime}} \approx\left(\left(X^{\mathrm{Ker} \tau}\right)^{\left|\Gamma^{\prime} \cdot i_{1}\right|}\right)^{\Gamma^{\prime}} \times \cdots \times\left(\left(X^{\mathrm{Ker} \tau}\right)^{\left|\Gamma^{\prime} \cdot i_{s}\right|}\right)^{\Gamma^{\prime}}
$$

by a permutation. Let $\Gamma^{\prime} \mid \Gamma_{l}^{\prime}=\gamma_{l, 1} \cdot \Gamma_{l}^{\prime} \amalg \cdots \amalg \gamma_{l, n(l)} \cdot \Gamma_{l}^{\prime}, \gamma_{l, 1}=1 . \quad$ A homeomorphism $f: X^{\tau^{-1}\left(\Gamma_{l}^{\prime}\right)} \rightarrow\left(\left(X^{\mathrm{Ker} \tau}\right)^{\left|\Gamma^{\prime} \cdot i_{l}\right|}\right)^{\Gamma^{\prime}}$ is defined by $f(x)=\left(\gamma_{l, 1} x, \cdots, \gamma_{l, n(l)} x\right)$. The inverse is given by the first projection. Therefore the desired homeomorphism is obtained.

LEMMA 3.9. The inclusion map

$$
\begin{aligned}
\mathscr{C}_{\infty}^{K(\tau)} \times\left([k]_{+}^{\tau(K)} \wedge\right. & \left.\left(\Sigma^{n \rho} X\right)^{K}\right) \rightarrow \mathscr{C}_{\infty}^{K(\tau)} \times\left(\left(\Sigma^{n_{\rho}} X\right)^{k}\right)^{K(\tau)}, \\
& (c, i \wedge y) \rightarrow(c, *, \cdots, *, y, *, \cdots, *)
\end{aligned}
$$

with $y$ in the $i$-th place, is $a(2 n(|G| H \mid+1)-1)$-equivalence.

Proof. It is sufficient to prove that $[k]_{+}^{\tau(K)} \wedge\left(\Sigma^{n \rho} X\right)^{K} \rightarrow\left(\left(\Sigma^{n \rho} X\right)^{k}\right)^{K(\tau)}$ is a $(2 n(|G / H|+1)-1)$-equivalence because $\mathscr{C}_{\infty}^{K(\tau)}$ is contractible. The composition of this inclusion and the homeomorphism of Lemma 3.8 is the map

$$
(*, i):[k]_{+}^{\tau(K)} \wedge\left(\sum^{n \rho} X\right)^{K} \longrightarrow\left(\left(\sum^{n \rho} X\right)^{\tau-1\left(\Gamma_{1}^{\prime}\right)} \times \cdots \times\left(\sum^{n \rho} X\right)^{\tau-1\left(\Gamma_{s}^{\prime}\right)}\right) \times\left(\left(\sum^{n \rho} X\right)^{K}\right)^{r},
$$

where $*$ is the constant map to the base point, $r=\left|[k]^{\tau(K)}\right|$ and

$$
i:[k]_{+}^{\tau(K)} \wedge\left(\Sigma^{n \rho} X\right)^{K} \approx\left(\Sigma^{n \rho} X\right)^{K} \vee \cdots \vee\left(\Sigma^{n \rho} X\right)^{K} \subset\left(\left(\Sigma^{n \rho} X\right)^{K}\right)^{r}
$$

is the canonical inclusion. $i$ is a $(4 n|G| K \mid-1)$-equivalence because $\operatorname{dim}\left(\sum^{n \rho}\right)^{K}$ $=2 n|G| K \mid . \quad \tau^{-1}\left(\Gamma_{l}^{\prime}\right) \leqq K$ because $[k-r]^{\tau(K)}=\phi$ and

$$
\operatorname{dim}\left(\Sigma^{n \rho}\right)^{\tau-1\left(\Gamma_{\imath}^{\prime}\right)}=2 n\left|G / \tau^{-1}\left(\Gamma_{\imath}^{\prime}\right)\right| \geqq 2 n(|G / K|+1) .
$$

Therefore $\left(\sum^{n \rho} X\right)^{\tau-1\left(\Gamma_{1}^{\prime}\right)} \times \cdots \times\left(\sum^{n \rho} X\right)^{\tau-1\left(\Gamma_{s}^{\prime}\right)}$ is $(2 n(|G / K|+1)-1)$-connected and $(*, i)$ is a $(2 n(|G / K|+1)-1)$-equivalence.

Put

$$
\begin{aligned}
& L(\tau)=\coprod_{\tau^{\prime} \in(\tau)} \mathscr{C}_{\infty}^{K\left(\tau^{\prime}\right)} \times\left(\left(\sum^{n_{\rho}} X\right)^{k}\right)^{K\left(\tau^{\prime}\right)}, \\
& M(\tau)=\coprod_{\tau^{\prime} \in(\tau)} \mathscr{C}_{\infty}^{K\left(\tau^{\prime}\right)} \times\left([k]_{+}^{\tau^{\prime}(K)} \wedge\left(\sum^{n_{\rho}} X\right)^{K}\right), \\
& N(\tau)=\coprod_{\tau^{\prime} \in(\tau)} \mathscr{C}_{\infty}^{K\left(\tau^{\prime}\right)}
\end{aligned}
$$


for $(\tau) \in \operatorname{Hom}(K, \Gamma) / \sim$. Let $\operatorname{Hom}(K, \Gamma) / \sim=\left\{\left(\tau_{1}\right), \cdots,\left(\tau_{s}\right)\right\}$. Then

$$
\begin{aligned}
& \left(\mathscr{C}_{\infty+} \bigwedge_{\Gamma}\left([k]_{+} \wedge \Sigma^{n \rho} X\right)\right)^{K}=\bigvee_{i=1}^{s}\left(M\left(\tau_{i}\right) / \Gamma\right) /\left(N\left(\tau_{i}\right) / \Gamma\right), \\
& \left(\mathscr{C}_{\infty+} \bigwedge_{\Gamma}\left(\Sigma^{n \rho} X\right)^{k}\right)^{K}=\bigvee_{i=1}^{s}\left(L\left(\tau_{i}\right) / \Gamma\right) /\left(N\left(\tau_{i}\right) / \Gamma\right),
\end{aligned}
$$

and $L(\tau) / \Gamma, M(\tau) / \Gamma, N(\tau) / \Gamma,(M(\tau) / \Gamma) /(N(\tau) / \Gamma)$ and $(L(\tau) / \Gamma) /(N(\tau) / \Gamma)$ are connected.

LEMMA 3.10.

i ) $(L(\tau) / \Gamma) /(M(\tau) / \Gamma)$ is $(2 n(|G / K|+1)-1)$-connected,

ii ) $(M(\tau) / \Gamma) /(N(\tau) / \Gamma)$ is $(2 n|G / K|-1)$-connected,

iii) $(L(\tau) / \Gamma) /(N(\tau) / \Gamma)$ is $(2 n|G / K|-1)$-connected.

Proof. i) $L(\tau)$ and $M(\tau)$ are $\Gamma$-free and the projection maps $L(\tau) \rightarrow$ $L(\tau) / \Gamma$ and $M(\tau) \rightarrow M(\tau) / \Gamma$ are principal $\Gamma$-bundles. The inclusion map $M(\tau)$ $\rightarrow L(\tau)$ is a $(2 n(|G| K \mid+1)-1)$-equivalence by Lemma 3.9 and $M(\tau) / \Gamma \rightarrow L(\tau) / \Gamma$ is so by the long exact sequence of homotopy groups for fibrations and five lemma. Then $\pi_{m}((L(\tau) / \Gamma) /(M(\tau) / \Gamma)) \cong \pi_{m}(L(\tau) / \Gamma, M(\tau) / \Gamma)=0,1 \leqq m \leqq 2 n(|G / K|$ $+1)-1$, by Blakers-Massey's theorem.

ii) is obtained by $(M(\tau) / \Gamma) /(N(\tau) / \Gamma)=\left(\bigvee_{\tau^{\prime} \in(\tau)} \mathscr{C}_{\infty_{+}} \wedge\left([k]_{+}^{\tau^{\prime}(K)} \wedge\left(\sum^{n \rho} X\right)^{K}\right)\right) / \Gamma$ $=\left(\sum^{n \rho}\right)^{K} \wedge\left(\bigvee_{\tau^{\prime} \in(\tau)} \mathscr{C}_{\infty+} \wedge\left([k]_{+}^{\tau^{\prime}(K)} \wedge X^{k}\right)\right) / \Gamma$.

iii) Each component of $L(\tau)$ is $(2 n|G / K|-1)$-connected by Lemma 3.8 and the proof is similar to i).

LEMMA 3.11. The inclusion map $(M(\tau) / \Gamma) /(N(\tau) / \Gamma) \rightarrow(L(\tau) / \Gamma) /(N(\tau) / \Gamma)$ is $a(2 n(|G / K|+1)-2)$-equivalence.

Proof. By Lemma 3.10, ii), iii) and the Blakers-Massey's theorem,

$$
\pi_{m}((L(\tau) / \Gamma) /(N(\tau) / \Gamma),(M(\tau) / \Gamma) /(N(\tau) / \Gamma)) \cong \pi_{m}((L(\tau) / \Gamma) /(M(\tau) / \Gamma))
$$

for $1 \leqq m \leqq 4 n|G| K \mid-2$ and it is equal to 0 for $1 \leqq m \leqq 2 n(|G| K \mid+1)-2$ by Lemma $3.10, \mathrm{i})$.

LEMMA 3.12. The inclusion map

$$
\bigvee_{i=1}^{s}\left(M\left(\tau_{i}\right) / \Gamma\right) /\left(N\left(\tau_{i}\right) / \Gamma\right) \rightarrow \bigvee_{i=1}^{s}\left(L\left(\tau_{i}\right) / \Gamma\right) /\left(N\left(\tau_{i}\right) / \Gamma\right)
$$

is a $(2 n(|G / K|+1)-2)$-equivalence.

Proof. The case $s=1$ is proved in Lemma 3.11. Assume the statement is proved for $s-1$. Put

$$
\begin{aligned}
& X_{1}=\bigvee_{i=1}^{s-1}\left(M\left(\tau_{i}\right) / \Gamma\right) /\left(N\left(\tau_{i}\right) / \Gamma\right), \quad X_{2}=\left(M\left(\tau_{s}\right) / \Gamma\right) /\left(N\left(\tau_{s}\right) / \Gamma\right), \\
& Y_{1}=\bigvee_{i=1}^{s-1}\left(L\left(\tau_{i}\right) / \Gamma\right) /\left(N\left(\tau_{i}\right) / \Gamma\right) \quad \text { and } \quad Y_{2}=\left(L\left(\tau_{s}\right) / \Gamma\right) /\left(N\left(\tau_{s}\right) / \Gamma\right) .
\end{aligned}
$$


Then $X_{i}$ and $Y_{i}, i=1,2$, are $(2 n|G| K \mid-1)$-connected and $\pi_{m}\left(X_{1} \vee X_{2}\right) \cong \pi_{m}\left(X_{1}\right)$ $+\pi_{m}\left(X_{2}\right)$ and $\pi_{m}\left(Y_{1} \vee Y_{2}\right) \cong \pi_{m}\left(Y_{1}\right)+\pi_{m}\left(Y_{2}\right)$ for $1 \leqq m \leqq 4 n|G / K|-2$. Therefore the statement is valid for $s$ by induction.

By the above lemma, (3.6) is an isomorphism for $2 n \geqq l$ and this completes the proof of Proposition 3.5.

Corollary 3.13. An equivariant stable wreath operation exists uniquely.

Proof of Theorem 2.3. We proved that an equivariant wreath operation satisfying (W1) and (W2) defines an equivariant stable wreath operation in (3.3). Conversely an equivariant stable wreath operation $\tilde{w}$ defines an equivariant wreath operation $w$ satisfying (W1) and (W2) by

$$
w(X): \mathscr{C}_{\infty+} \bigwedge_{\Gamma} X^{k} \subset \operatorname{Tel} \Omega^{n \rho}\left(\mathscr{C}_{\infty+} \bigwedge_{\Gamma}\left(\Sigma^{n \rho} X\right)^{k}\right) \stackrel{\tilde{w}(X)}{\longrightarrow} Q_{G}(X) .
$$

$w$ satisfies (W2) clearly. To prove that it satisfies (W1) we show the diagram

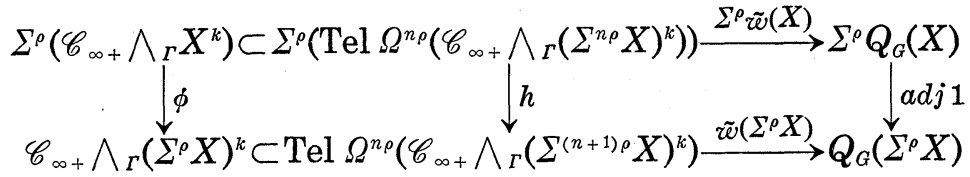

is commutative in $\mathscr{T}_{G *}$, where

$$
h(v \wedge[f \wedge t])=\left[f^{\prime} \wedge t\right], v \in \Sigma^{\rho},[f \wedge t] \in \operatorname{Tel} \Omega^{n_{\rho}}\left(\mathscr{C}_{\infty_{+}} \wedge_{\Gamma}\left(\Sigma^{n_{\rho}} X\right)^{k}\right)
$$

and $f^{\prime}: \Sigma^{n \rho} \rightarrow \mathscr{C}_{\infty+} \bigwedge_{\Gamma}\left(\sum^{(n+1) \rho} X\right)^{k}$ is defined by $f^{\prime}\left(v^{\prime}\right)=\phi\left(v \wedge f\left(v^{\prime}\right)\right)$. The left square is commutative by definition. Put $\tilde{w}^{\prime}(X)=\Omega^{\circ} \tilde{w}\left(\Sigma^{\rho} X\right) \circ(a d j h)$. It is easily checked that $\tilde{w}^{\prime}$ is an equivariant stable wreath operation. Therefore $\tilde{w}^{\prime}=\tilde{w}$ and the right square of the above diagram is commutative in $\mathscr{T}_{G * \cdot}$

The correspondences $w \mapsto \tilde{w}$ of (3.3) and $\tilde{w} \mapsto w$ of (3.14) are inverses to each other and equivariant wreath operation satisfying (W1) and (W2) exists uniquely. Then an equivariant transfer on $\operatorname{Cov}_{G}^{\Gamma}$ exists uniquely by Lemmas 2.8, 2.9 and 2.10 .

The equivariant transfer of $p:(X, A) \rightarrow(Y, B) \in \operatorname{Cov}_{G}^{\Gamma}$ may be described explicitly as the following composition.

$$
t(p):(Y, B) \stackrel{T / \Gamma}{\longrightarrow} \mathscr{C}_{\infty+} \bigwedge_{\Gamma}(X / A)^{k} \subset \mathscr{C}_{\infty+} \bigwedge_{\Gamma} Q_{G}(X / A)^{k} \stackrel{\theta_{\infty, k}}{\longrightarrow} Q_{G}(X / A) .
$$

By a routine argument we obtain

Proposition 3.15. i) Let $p:(X, A) \rightarrow(Y, B)$ be a k-fold G-covering, $(X, A)=\left(X_{1}, A_{1}\right) \amalg\left(X_{2}, A_{2}\right)$ and $p_{i}=\left.p\right|_{\left(x_{i}, A_{i}\right)}$ are $k_{i}$-fold $G$-coverings, $i=1,2$. The 
diagram

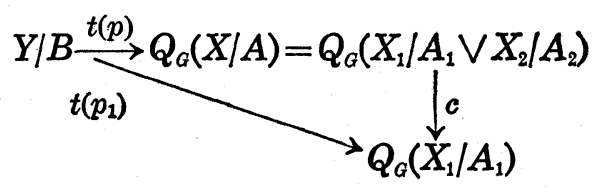

is commutative in $\mathscr{T}_{G *}$, where $c$ is induced by the collapsing map $X_{1} / A_{1} \vee X_{2} / A_{2}$ $\rightarrow X_{1} / A_{1}$.

ii) Let $p_{1}:(X, A) \rightarrow(Y, B)$ be a $k_{1}$-fold $G$-covering and $p_{2}:(Y, B) \rightarrow(W, D)$ $a k_{2}$-fold $G$-covering. The diagram

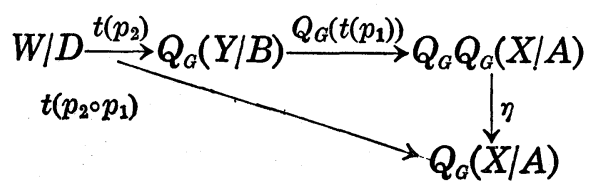

is commutative in $\mathscr{T}_{G *}$, where $\eta$ is the canonical infinite loop G-map.

\section{§4. The equivariant Becker-Gottlieb transfer and Atiyah transfer}

Let $\pi: X \rightarrow Y$ be a fiber bundle with $G$-action in the sense of [14] with compact fiber over a finite $G$-complex $Y$. The equivariant Becker-Gottlieb transfer $\tau(\pi): \Sigma^{V}\left(Y_{+}\right) \rightarrow \Sigma^{V}\left(X_{+}\right)$for $\pi$ is defined by Nishida [12]. Especially, $k$-fold $G$-covering $p: X \rightarrow Y$ over a finite $G$-complex $Y$ has such an equivariant Becker-Gottlieb transfer $\tau(p)$. We show that $\tau(p)$ is an adjoint of $t(p)$. Let $\tilde{p}: Z \rightarrow Y$ be a principal $(G, \Gamma)$-bundle associated with $p$. Define $Z \times_{\Gamma}[k] \subset$ $Z \times{ }_{\Gamma} C^{k}$ by $[z, i] \rightarrow[z, 0, \cdots, 0,1,0, \cdots, 0]$ with 1 in the $i$-th place. $Z \times{ }_{\Gamma} C^{k}$ can be embedded in $Y \times V$ as a subbundle for a representation $V$ of $G$ because $Z \times{ }_{\Gamma} C^{k}$ is a complex $G$-vector bundle over a finite $G$-complex $Y$. Let $\alpha$ be the composition map $X \approx Z \times{ }_{\Gamma}[k] \subset Z \times{ }_{\Gamma} C^{k} \subset Y \times V \stackrel{p r_{2}}{\longrightarrow} V$. Then there is an embedding $i: X \times D V \rightarrow Y \times D V$ defined by $i(x, v)=(p(x), \alpha(x)+\varepsilon v)$ for small $\varepsilon>0$. $\quad \tau(p)$ is defined by

$$
\tau(p)(v \wedge y)=\left\{\begin{array}{cl}
v^{\prime} \wedge x, & \text { if } y=p(x) \text { and } v=\alpha(x)+\varepsilon v^{\prime} \\
*, & \text { otherwise. }
\end{array}\right.
$$

$V$ may be replaced by $n \rho$ for large $n$. Let

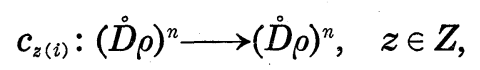

be the map defined by $c_{z(i)}(v)=\alpha(z(i))+\varepsilon v$, where we identify $\left(\dot{D}_{\rho}\right)^{n}$ with $\dot{D}(n \rho)$. The map $d: Z \rightarrow \mathscr{C}_{\infty+}(k ; G)$ defined by $d(z)=\left(c_{z(1)}, \cdots, c_{z(k)}\right)$ is a $G \times \Gamma$-map and we use it to define $t(p)$. Let $y \in Y$ correspond to $[z]$ by $Y \approx_{G} Z \mid \Gamma$. Then 
$t(p)(y): \Sigma^{n \rho} \rightarrow \Sigma^{n \rho} \wedge X_{+}$is defined by

$$
t(p)(\hat{y})(i)=\left\{\begin{array}{cl}
c_{z(i)}^{-1}(v) \wedge z(i), & \text { if } v \in \operatorname{Im} c_{z(i)}, \\
*, & \text { otherwise. }
\end{array}\right.
$$

Therefore the adjoint of $t(p)$ is equal to $\tau(p)$.

Next we observe that the Atiyah transfer for $K_{G}$-theory is the induced homomorphism in $K_{G}$-theory induced from the adjoint of the equivariant transfer. Let $\tilde{h}_{G}^{*}$ be a reduced $G$-cohomology theory represented by an $\Omega$ $G$-spectrum $E=\left\{E_{n}\right\}, E_{n} \simeq{ }_{G} \Sigma^{\circ} E_{n+1}$, (Araki-Murayama [1], Theorem 3.4). Let $p: X \rightarrow Y$ be as above. Then $t(p): Y_{+} \rightarrow \Omega^{m \rho} \Sigma^{m \rho}\left(X_{+}\right)$for some $m$. Define $\operatorname{tr}[\alpha]$ $\in\left[Y_{+}, E_{n}\right]^{G}$ for $[\alpha] \in\left[X_{+}, E_{n}\right]^{G}$ as the composition

$$
Y_{+} \stackrel{T / \Gamma}{\longrightarrow} \mathscr{C}_{m}(k ; G)_{+} \wedge_{\Gamma}\left(X_{+}\right)^{k} \stackrel{1_{+} \wedge_{\Gamma} \alpha^{k}}{\longrightarrow} \mathscr{C}_{m}(k ; G)_{+} \wedge_{\Gamma}\left(E_{n}\right)^{k} \stackrel{\theta_{m, k}}{\longrightarrow} E_{n} .
$$

By the same proof as in [6], Proposition 1.4, we get

Lemma 4.1. The following diagram is commutative.

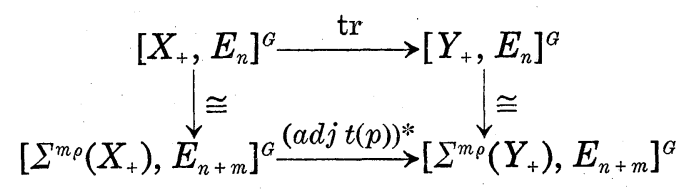

Let $\pi: E \rightarrow X$ be a complex $G$-vector bundle and $p_{*} E$ be the direct image bundle (Atiyah [2], p. 26). The fiber $\left(p_{*} E\right)_{y}$ is defined as the direct sum $\oplus_{x} E_{x}$ where $x \in p^{-1}(y) . \quad G$ acts on $p_{*} E$ obviously and this induces the Atiyah transfer $p_{*}: K_{G}(X) \rightarrow K_{G}(Y)$. Let $B U_{\theta}$ be the 0 -th term of $\Omega$-G-spectrum representing the reduced $K_{G}$-theory. To show $(\operatorname{adj} t(p))^{*}=p_{*}$, it is sufficient to prove that $p_{*}$ is represented by $\operatorname{tr}[\alpha]$ when $\pi$ is represented by $\alpha: X \rightarrow B U_{G} . \quad 1 \times \pi^{k}: \mathscr{C}_{\infty}(k ; G) \times E^{k} \rightarrow \mathscr{C}_{\infty}(k ; G) \times X^{k}$ is a $G \times \Gamma$-vector bundle and the base space is $\Gamma$-free. Therefore $1 \times{ }_{\Gamma} \pi^{k}$ is a $G$-vector bundle. An isomorphism $\phi:(T / \Gamma)^{*}\left(1 \times_{\Gamma} \pi^{k}\right) \rightarrow p_{*} E$ is defined by $\phi\left([z],\left[d(z), e_{1}, \cdots, e_{k}\right]\right)=$ $\left(e_{1}, \cdots, e_{k}\right) \in\left(p_{*} E\right)_{y}, y=[z]$, and hence $(\operatorname{adj} t(p))^{*}=p_{*}$.

\section{References}

[1] S. Araki and M. Murayama, $G$-homotopy types of $G$-complexes and representation of G-cohomology theories, Publ. Res. Inst. Math. Sci., 14 (1978), 203-222.

[2] M. F. Atiyah, Characters and cohomology of finite groups, Publ. Math. I.H.E.S., 9 (1961), 23-64.

[ 3 ] J. C. Becker and D. H. Gottlieb, The transfer maps and fibre bundles, Topology, 14 (1975), 1-12.

[ 4 ] J. M. Boardman and R. M. Vogt, Homotopy everything $H$-spaces, Bull. Amer. Math. Soc., 74 (1968), 1117-1122. 
[5] G. E. Bredon, Equivariant Cohomology Theories, Lecture Notes in Math., 34, Springer, 1967.

[6] D. S. Kahn and S. B. Priddy, Applications of the transfer to stable homotopy theory, Bull. Amer. Math. Soc., 78 (1972), 981-987.

[ 7 ] D. S. Kahn and S. B. Priddy, The transfer and stable homotopy theory, Math. Proc. Camb. Phil. Soc., 83 (1978), 103-111.

[ 8 ] S. Kakutani and M. Murayama, Equivariant duality, reducibility and coreducibility, to appear.

[ 9 ] J. P. May, The Geometry of Iterated Loop Spaces, Lecture Notes in Math., 271, Springer, 1972.

[10] M. Murayama, Equivariant S-duality, unpublished.

[11] U. Namboodiri, Equivariant vector fields on spheres, Thesis, Univ. of Chicago, 1982.

[12] G. Nishida, The transfer homomorphism in equivariant generalised cohomology theories, J. Math. Kyoto Univ., 18-3 (1978), 435-451.

[13] F. W. Roush, Transfer in generalised cohomology theories, Thesis, Princeton Univ., 1971.

[14] T. tom Dieck, Faserbündel mit Gruppenoperation, Arch. Math., 20 (1969), 136143.

[15] S. Waner, Equivariant homotopy theory and Milnor's theorem, Trans. Amer. Math. Soc., 258 (1980), 351-358.

\author{
DepartMent OF MATHEMatics \\ OSAKa City UNIVERSity \\ SUMIYOSHI-KU, OSAKA 558 \\ JAPAN
}

